the numbers which Epstein, in his recent article published in the Archiv der Mathematik und Physik, calls primitive roots of $m$ are those which correspond to the operators of highest order in the group of isomorphisms of the cyclic group of order $m$, and hence the determination of the number of such primitive roots is a very special case of the determination of the number of operators of a given order in an abelian group. In the present paper special attention is paid to the group of isomorphisms of the holomorph of the cyclic group of order $2^{m}$, and one of the most important results is stated as follows: The group of isomorphisms of the holomorph of the cyclic group of order $2^{m}$ is the direct product of the group of order 2 and the group of cogredient isomorphisms of the double holomorph of the cyclic group of order $2^{m}$. This paper will appear in the Transactions.

14. In this paper Professor Davis studies the connection between his theory of colored imaginaries and the pole and polar theory of the cubic curve. If $f(x, y, z)=0$ is the equation of the cubic, $\Delta^{\prime}(x, y, z)$ the first polar of $x^{\prime}, y^{\prime}, z^{\prime}$ with regard to $f(x, y, z)$, while $\Delta^{\prime \prime}$ is the polar of $x^{\prime \prime}, y^{\prime \prime}, z^{\prime \prime}$, then when we write $x=x^{\prime}+i x^{\prime \prime}$, etc., we get $f\left(x^{\prime}, y^{\prime}, z^{\prime}\right)=\Delta^{\prime}\left(x^{\prime \prime}, y^{\prime \prime}, z^{\prime \prime}\right)$ and $f\left(x^{\prime \prime}, y^{\prime \prime}, z^{\prime \prime}\right)=\Delta^{\prime \prime}\left(x^{\prime}, y^{\prime}, z^{\prime}\right)$. It is upon the basis of these two equations that the paper is built.

O. D. KellogG, Secretary of the Section.

\title{
NOTE ON THE COMPOSITION OF FINITE ROTATIONS ABOUT PARALLEL AXES.
}

BY PROFESSOR ALEXANDER ZIWET.

1. IT is well known that the succession of two finite rotations of a rigid plane figure in its plane (or, what amounts to the same, of a rigid body about parallel axes), say a rotation of angle $\theta^{\prime}$ about a point $O^{\prime}$ followed by a rotation $\theta^{\prime \prime}$ about $O^{\prime \prime}$, is equivalent to a single rotation of angle $\theta=\theta^{\prime}+\theta^{\prime \prime}$ about a point $O$. The center $O$ is found as the intersection of the lines obtained by turning $O^{\prime} O^{\prime \prime}$ about $O^{\prime}$ through an angle $-\frac{1}{2} \theta^{\prime}$ and $O^{\prime \prime} O^{\prime}$ about $O^{\prime \prime}$ through $+\frac{1}{2} \theta^{\prime \prime}$.

As a clockwise rotation of angle $\phi$ is equivalent to a counterclockwise rotation of angle $2 \pi-\phi$, the angles of rotation can 
all be made of the same sense, say counterclockwise; and as a rotation of angle $\phi+2 \kappa \pi$ is equivalent to the rotation of angle $\phi$, the angles can be confined to values between 0 and $2 \pi$.

The construction of the point $O$ is therefore always possible unless $\theta=\theta^{\prime}+\theta^{\prime \prime}$ is $\equiv 0(\bmod 2 \pi)$. In this limiting case the center lies at infinity, and the resultant displacement is a translation whose vector is readily determined.

2. When there are more than two successive rotations, the resultant of the first and second rotations can be compounded by the same method with the third rotation, and so on. It is apparent that the angle of the final resultant rotation is the sum of the angles of the given rotations, but the construction of the center becomes rather complicated. It is the object of the present note to indicate a convenient method for finding this center, by applying vector methods systematically.

3. A single rotation, $\theta^{\prime}$ about $O^{\prime}$ (Fig. 1 ), carries any point $A$ of the rigid figure from the initial position $A_{0}$ to the final

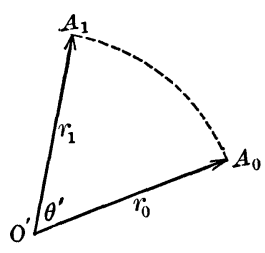

FIG. 1.

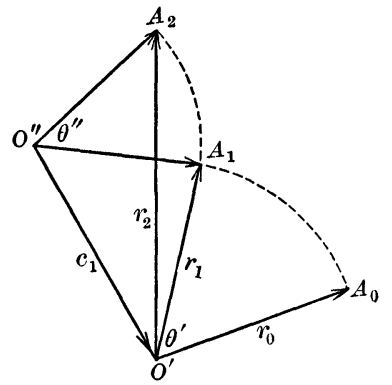

FIG. 2.

position $A_{1}$. Taking the fixed point $O^{\prime}$ as origin and putting $A_{0}-O^{\prime}=\mathrm{r}_{0}, A_{1}-O^{\prime}=\mathrm{r}_{1}$, we have the vector equation

$$
\mathbf{r}_{1}=e^{i \theta^{\prime}} \mathbf{r}_{0}
$$

which means that the operator $e^{i \theta^{\prime}}$ applied to the vector $\mathbf{r}_{0}$ turns it in the plane through the angle $\theta^{\prime} .{ }^{*}$ ) This is of course merely another form of stating the ordinary method of complex numbers.

Two successive rotations, $\theta^{\prime}$ about $O^{\prime}$ and $\theta^{\prime \prime}$ about $O^{\prime \prime}$ (Fig. 2), carry the point $A$ from $A_{0}$ to $A_{1}$ and from $A_{1}$ to $A_{2}$. With $O^{\prime}-O^{\prime \prime}=\mathrm{c}_{1}, \quad A_{2}-O^{\prime}=\mathrm{r}_{2}$ we have $A_{1}-O^{\prime \prime}=\mathrm{r}_{1}+\mathrm{c}_{1}$,

${ }^{*}$ G. Peano, Gli elementi di calcolo geometrico, Torino, 1891, p. 19. 
$A_{2}-O^{\prime \prime}=\mathrm{r}_{2}+\mathrm{c}_{1}$, whence

$$
\mathbf{r}_{2}+\mathbf{c}_{1}=e^{\imath \theta^{\prime \prime}}\left(\mathbf{r}_{1}+\mathbf{c}_{1}\right)
$$

or, replacing $\mathbf{r}_{1}$ by its value from the preceding equation,

$$
\mathbf{r}_{2}=e^{i\left(\theta^{\prime \prime}+\theta^{\prime}\right)} \mathbf{r}_{0}+e^{i \theta^{\prime \prime}} \mathbf{c}_{1}-\mathbf{c}_{1} .
$$

In the case of three successive rotations, $\theta^{\prime}$ about $O^{\prime}, \theta^{\prime \prime}$ about $O^{\prime \prime}, \theta^{\prime \prime \prime}$ about $O^{\prime \prime \prime}$, putting $O^{\prime \prime}-O^{\prime \prime \prime}=\mathrm{c}_{2}, A_{3}-O^{\prime}=\mathrm{r}_{3}$ we have

$$
\mathrm{r}_{3}+\mathrm{c}_{1}+\mathrm{c}_{2}=e^{i \theta^{\prime \prime \prime}}\left(\mathrm{r}_{2}+\mathrm{c}_{1}+\mathrm{c}_{2}\right) \text {, }
$$

whence eliminating $\mathbf{r}_{2}$ we find

$$
\mathbf{r}_{3}=e^{i\left(\theta^{\prime \prime \prime}+\theta^{\prime \prime}+\theta^{\prime}\right)} \mathbf{r}_{0}+e^{i\left(\theta^{\prime \prime \prime}+\theta^{\prime \prime}\right)} \mathbf{c}_{1}+e^{i \theta^{\prime \prime \prime}} \mathbf{c}_{2}+\mathbf{c}_{3}
$$

where $\mathrm{c}_{3}=-\mathrm{c}_{1}-\mathrm{c}_{2}=O^{\prime \prime \prime}-O^{\prime}$.

Similarly we find for four successive rotations, $\theta^{\prime}$ about $O^{\prime}$, $\cdots, \theta^{\text {iv }}$ about $O^{\text {iv }}$

$$
\mathbf{r}_{4}=e^{i\left(\theta^{\mathrm{iv}}+\theta^{\prime \prime \prime}+\theta^{\prime \prime}+\theta^{\prime}\right)} \mathbf{r}_{0}+e^{i\left(\dot{\theta}^{\mathrm{i}}+\theta^{\prime \prime \prime}+\theta^{\prime \prime}\right)} \mathbf{c}_{1}+e^{i\left(\theta^{\mathrm{iv}}+\theta^{\prime \prime \prime}\right)} \mathbf{c}_{2}+\mathbf{c}^{i \theta^{\mathrm{iv}}} \mathbf{c}_{3},+\mathbf{c}_{4},
$$

where $c_{1}+c_{2}+c_{3}+c_{4}=0$. The vectors $c_{1}, \cdots, c_{4}$ are equal to the sides of the quadrilateral formed by the centers $O^{\prime}, \ldots, O^{\text {iv }}$; these centers are points of the fixed plane, not of the moving figure. The initial and final radii vectores $\mathbf{r}_{0}, \mathbf{r}_{4}$ of $A$ are drawn from $O^{\prime}$. If any other point $Q$ of the plane were taken as origin, it would only be necessary to replace $r_{0}, r_{4}$ by $r_{0}^{\prime}-q$, $\mathbf{r}_{4}^{\prime}-\mathbf{q}$, where $\mathbf{r}_{0}^{\prime}, \mathbf{r}_{4}^{\prime}$ are the radii vectores of $A$ drawn from $Q$, and $\mathrm{q}=O^{\prime}-Q$.

4. In the expression found for $\mathbf{r}_{4}$, all terms after the first are independent of the particular point $A$ of the figure. Their sum represents a vector $s_{4}$ which can be written

$$
\mathbf{s}_{4}=e^{i \theta^{i \mathrm{v}}}\left(e^{i \theta^{\prime \prime \prime}}\left(e^{i \theta^{\prime \prime}} \mathbf{c}_{1}+\mathbf{c}_{2}\right)+\mathbf{c}_{3}\right)+\mathbf{c}_{4} .
$$

This form indicates the most convenient way of constructing the vector $\mathrm{s}_{4}$ (Fig. 3): turn $O^{\prime}-O^{\prime \prime}=\mathrm{c}_{1}$ about $O^{\prime \prime}$ through $\theta^{\prime \prime}$ and add $\mathrm{c}_{2}$; then turn the sum so obtained about $O^{\prime \prime \prime}$ through $\theta^{\prime \prime \prime}$ and add $c_{3}$; finally turn the vector so obtained about $O^{\text {iv }}$ through $\theta^{\text {iv }}$ and add $\mathrm{c}_{4}$; this gives the vector $\mathrm{s}_{4}=O_{4}-O^{\prime}$ which evidently represents the displacement of that point of the rigid figure which originally coincided with $O^{\prime}$. 
Denoting the angle of the resultant rotation by $\theta$, so that $\theta \equiv \theta^{\prime}+\theta^{\prime \prime}+\theta^{\prime \prime \prime}+\theta^{\text {iv }}(\bmod 2 \pi)$ and introducing the vector $\mathrm{s}_{4}$, we have the simple result

$$
\mathbf{r}_{4}=e^{i \theta} \mathbf{r}_{0}+\mathbf{s}_{4} .
$$

5. It is obvious that in the case of $n$ successive rotations we have similarly

$$
\mathbf{r}_{n}=e^{i \theta} \mathbf{r}_{0}+\mathbf{s}_{n}
$$

where $\theta \equiv \theta^{\prime}+\theta^{\prime \prime}+\cdots+\theta^{(n)}$ and

$$
\mathbf{s}_{n}=e^{i \theta(n)}\left\{\cdots\left[e^{i \theta^{\prime \prime \prime}}\left(e^{i \theta^{\prime \prime}} \mathbf{c}_{1}+\mathbf{c}_{2}\right)+\mathbf{c}_{3}\right]+\cdots+\mathbf{c}_{n-1}\right\}+\mathbf{c}_{n} .
$$

Thus, the final radius vector of any point of the rigid figure is found by turning its initial radius vector about $O^{\prime}$ through an

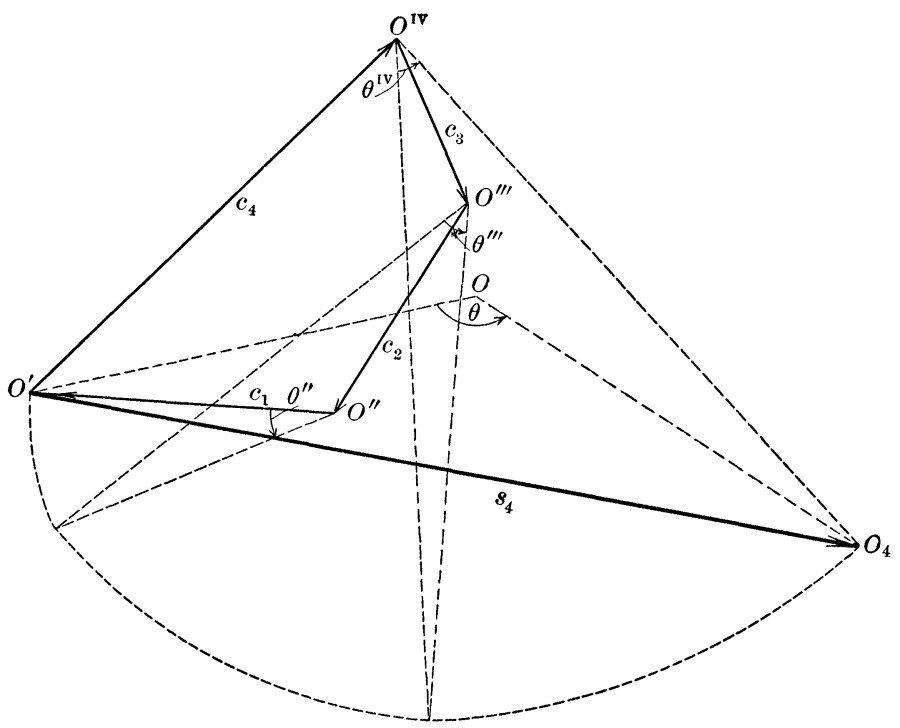

FIG. 3.

angle $\theta \equiv \theta^{\prime}+\theta^{\prime \prime}+\cdots+\theta^{(n)}$ and adding to it the vector $\mathbf{s}_{n}$. In other words, the resultant displacement is resolved into the rotation of angle $\theta$ about $O^{\prime}$ and the translation $\mathbf{s}_{n}$ of $O^{\prime}$.

The center $O$ of the equivalent single rotation being a fixed point its radius vector $r$ is found by putting $r_{n}=r_{0}=r$ which 
giv

$$
\left(1-e^{i \theta}\right) \mathbf{r}=\mathbf{s}_{n}, \quad \text { or } \quad \mathbf{r}=\frac{1}{2 \sin \frac{1}{2} \theta} e^{i(\pi-\theta) / 2} \mathbf{s}_{n}
$$

This means that the point $O$ is the vertex of the isosceles triangle whose base is $O^{\prime} O_{n}$ and whose angle at $O$ is $\theta$ if $\theta<\pi$ and is $2 \pi-\theta$ if $\theta>\pi$. In the former case the sense $O^{\prime} O_{n} O$ is positive, in the latter it is negative. If $\theta=\pi$, the resultant displacement is a reversal (Umwendung) about the midpoint of $O^{\prime} O_{n}$. If $\theta \equiv 0(\bmod 2 \pi)$ the resultant displacement reduces to the translation $\mathrm{s}_{n}$.

If, in particular, the angles of rotation $\theta^{\prime \prime}, \theta^{\prime \prime \prime}, \cdots, \theta^{(n)}$ are equal, respectively, to the exterior angles of the polygon of centers at $O^{\prime \prime}, O^{\prime \prime \prime}, \cdots, O^{(n)}$, the vector $\mathrm{s}_{n}$ has, as appears at once from its construction (Fig. 3), the direction and sense of $\mathbf{c}_{n}$ and a length equal to the perimeter of the polygon $O^{\prime} O^{\prime \prime} \ldots O^{(n)}$. If, in addition, $\theta^{\prime}$ is equal to the exterior angle at $O^{\prime}$ and the polygon is convex, the resultant rotation is zero, and the resultant displacement reduces to the translation $\mathbf{s}_{n}$.

ANN ARBor, December, 1907.

\section{ON AN INTEGRAL APPEARING IN PHOTOMETRY.}

BY PROFESSOR A. S. CHESSIN.

(Read before the Southwestern Section of the American Mathematical Society, November 30, 1907.)

In the course of research on the variation of the intensity of illumination, Mr. E. P. Hyde found it necessary to evaluate the integral

$$
\int \frac{i \cos \phi \cos \theta}{r^{2}} d S
$$

taken over a part of the surface of a cylinder. $\dagger$ Having encountered some difficulties on the mathematical side of the problem, Mr. Hyde requested the author to compute the value of this integral. As this computation contains some features of interest to mathematicians the solution is here presented

† "Talbot's law as applied to the rotating sectored disc," by E. P. Hyde, Bulletin of the U. S. Bureau of Standards, vol. 2 , No. 1. 